community pharmacies. This service is available to people who are injecting drugs but not on opiate substitution therapy. We surveyed operational delivery network (ODN) staff across England to understand the current implementation of the NHSE advanced service.

Method Service leads and key stakeholders within each ODN in England were identified by the Hepatitis C Trust. The survey was designed using Microsoft Forms. It was advertised through the NHSE HCV newsletter and distributed by HCV Action. Results were analysed using Microsoft Excel.

Results We received 22 responses from 20 out of 23 ODNS $(87 \%)$. We analysed the results per ODN $(n=20)$. In two ODNs there were two respondents, if the answers matched we included the answer once; if one respondent had a definitive answer and the other an unknown response, the definitive answer was used; and if there were contradictory answers, the response from this ODN was excluded.

Fourteen (70\%) ODNs had met with a representative from the local pharmaceutical committee and $95 \%$ of ODNs had pharmacies that had registered interest in the advanced service, with more than ten pharmacies registering interest in the majority of ODNs $(12(63 \%))$. Four (20\%) ODNs had arranged additional training for pharmacies. Fourteen $(70 \%)$ respondents were aware of their referral pathway. Two (10\%) ODNs reported that the pharmacist would perform a dry blood spot test to check RNA status if a client was antibody positive. Only three (15\%) ODNs reported that testing had started in their region, despite eight (40\%) ODNs reporting a prior pilot of pharmacy-based HCV testing.

$16 / 22(73 \%)$ of respondents wanted further guidance on the implementation of the service, this included guidance with the initial set up and how to effectively communicate between clinicians and pharmacists. Qualitative feedback about the service was varied, however, a consistent theme was the need to expand the eligible population for the service.

Conclusions The NHSE HCV antibody testing service has started in a small number of community pharmacies in a minority of ODNs. Many ODNs have had prior successful pilot testing programme. There is therefore an opportunity to learn from these experiences to support the implementation of the national service.

\section{P032 INCREASING BURDEN OF ALCOHOL-RELATED LIVER DISEASE IN THE UK ASSOCIATED WITH THE CORONAVIRUS PANDEMIC}

\begin{abstract}
${ }^{1,2}{ }^{2}$ Ashwin Dhanda*, ${ }^{3}$ Michael Allison, ${ }^{4}$ Keith Bodger, ${ }^{5}$ Ewan Forrest, ${ }^{4}$ Steve Hood, ${ }^{6}$ Alastair MacGilchrist, ${ }^{7}$ Steven Masson, ${ }^{8}$ Richard Parker, ${ }^{6}$ Ken Simpson, ${ }^{9}$ Nikhil Vergis, ARLD SIG service evaluation group. 'University Hospitals Plymouth NHS Trust, Plymouth, UK; ${ }^{2}$ University of Plymouth, Plymouth, UK; ${ }^{3}$ Cambridge University Hospitals NHS Foundation Trust, Cambridge, UK; ${ }^{4}$ Liverpool University Hospitals NHS Foundation Trust, Liverpool, UK; ${ }^{5}$ Glasgow Royal Infirmary, Glasgow, UK; ${ }^{6}$ Royal Infirmary of Edinburgh, UK; ${ }^{7}$ Newcastle Hospitals NHS Foundation Trust, Newcastle, UK; ${ }^{8}$ Leeds Teaching Hospitals NHS Trust, Leeds, UK; ${ }^{9}$ mperial College Healthcare NHS Trust, London, UK
\end{abstract}

\subsection{6/gutjnl-2021-BASL.4}

During the first UK national coronavirus pandemic lockdown (Mar-Jul 2020), alcohol sales increased 30\% in supermarkets. Surveys reported that $20 \%$ of people increased their alcohol consumption and numbers of high-risk drinkers increased by $13 \%$. Post-lockdown, clinicians noted high numbers of alcohol-related liver disease (ArLD)-related admissions. We hypothesised that greater alcohol consumption in high-risk drinkers contributed to this increase. We conducted a national service evaluation to document the number and severity of unplanned ArLD hospital admissions pre- and post-lockdown.

We performed a retrospective service evaluation in $28 \mathrm{UK}$ hospitals of all unplanned admissions during a one-week period in August 2019 and the same period in August 2020. The protocol was approved by the lead site's Clinical Audit Department and registered at participating sites. We applied a validated coding algorithm that more accurately identifies ArLD admissions than using only ArLD codes in the primary diagnosis. ${ }^{1}$ Eligible cases were manually reviewed and data extracted into a pre-designed collection tool. Data collected included demographics, diagnosis, alcohol use and liver disease severity scores, which were compared between evaluation periods.

There was an $18 \%$ absolute increase in unplanned hospital admissions for patients with ArLD in the evaluation period in 2020 compared to 2019 (263 vs 223). Demographics were similar between the two periods (mean age 55;37\% female). In-hospital mortality was similar $(9.0 \%$ vs $7.2 \%)$ and there were no differences between proportions of patients with complications of liver disease including variceal bleeding and alcoholic hepatitis. Patients in both evaluation periods had similar severity of liver disease with mean Child Pugh score of 8 and MELD 14. Those with alcoholic hepatitis had mean MELD 20 (SD 7.5) and discriminant function 90 (SD 70).

In the post-lockdown period, there were more active alcohol drinkers (151 vs $196 ; 75 \%$ vs $68 \%$ ) than pre-lockdown. Mean consumption per patient was higher (154 vs 127 units alcohol/week; $\mathrm{p}=0.02)$. More patients reported drinking spirits post- vs pre-lockdown ( $31 \%$ vs $22 \%$; $=0.06)$.

This national service evaluation demonstrates an increase in unplanned ArLD hospital admissions post-lockdown with patients reporting heavier alcohol use. Although there were no differences in clinical presentations or outcomes, these patients have advanced liver disease with high short-term mortality. These data suggest the pandemic has disproportionately affected high-risk drinkers and demonstrate the heavy burden of ArLD in the UK. There is an ongoing need to develop long-term strategies to improve these patients' outcomes.

\section{REFERENCE}

1. Kallis, et al. Aliment Pharm Therap 2020;52:182-95.

\section{P033 DELIVERY OF LIVER SERVICES WITHIN PRIMARY CARE CAN IMPROVE TREATMENT OUTCOMES IN PERSONS EXPERIENCING HOMELESSNESS}

${ }^{1}$ Jennifer Hannon*, ${ }^{1}$ Helen Morgan, ${ }^{2}$ Carla Boreland, ${ }^{2}$ Aurelia Polocenkaite, ${ }^{2}$ Jack Jerjian, ${ }^{1}$ Fiona Jones, ${ }^{3} J o h n$ Gibbons, ${ }^{2}$ Keith McDonald, ${ }^{2}$ Alexandre Wilkins, ${ }^{2}$ Tanya O'Brien, ${ }^{2}$ Mary Hickey, ${ }^{2}$ Paul O'Reilly, ${ }^{1}$ Matthew Foxton. 'Chelsea and Westminster Hospital, London, UK; ' 2 r Hickey's Surgery, London, UK; ${ }^{3}$ Find and Treat Team, University College Hospitals, London, UK

\subsection{6/gutjnl-2021-BASL.42}

Background Persons experiencing homelessness (PEH) often have complex health needs compounded by difficulty accessing healthcare. Liver disease is the third commonest cause for death in PEH after accidents and suicides. Some studies have identified chronic hepatitis $\mathrm{C}(\mathrm{CHC})$ in up to $50 \%$ of this population. Alcohol disorders are also extremely common. 
Methods From June 2018, a weekly Hepatology clinic was co-located with a primary care clinic serving a population of 2,500 PEH. Blood borne virus testing, near patient HCV RNA testing, transient elastography and anti-viral drug dispensing for Hepatitis $\mathrm{B}$ and $\mathrm{C}$ was introduced. All patients with HCV were treated in line with national guidelines.

Results Between the period of June 2018 and November 2020, 326 patients were reviewed within the Hepatology clinic. A total of 1,236 appointments were offered with 632 attendances $(51.1 \%)$.

241 patients were referred due to a positive $\mathrm{HCV}$ Ab test. 193 were RNA positive (80\%), 30 RNA negative (12.4\%) and 19 had unknown HCV RNA status. Transient elastography was performed on 138 with 31 having advanced fibrosis.

Treatment was initiated on 101/193 HCV RNA positive patients. 93 patients were receiving opiate substitution therapy. $65 \%$ had a co-existing mental health diagnosis whilst $24 \%$ had a significant alcohol intake, $2 \%$ were co-infected with Hepatitis B and C and 3\% were co-infected with both Hepatitis $\mathrm{C}$ and HIV. The genotypes were $44 \mathrm{G} 1 \mathrm{a} ; 3 \mathrm{G} 1 \mathrm{~b} ; 6 \mathrm{G} 2$; 34 G3; 1 G4 and 13 unknown.

There is a high rate of sustained virological response (SVR) being achieved with 61 patients having achieved SVR (82\%). 13 patients needed to re-start treatment. 37 have SVR 12 pending. 3 patients have relapsed.

Of the remaining 92 known RNA positive patients within the clinic, 40 identified within our service have been treated elsewhere during the peak of the Covid-19 pandemic. 24 SVR blood tests were performed for the patients treated in other locations. 21 patients are approved to start treatment. 9 are awaiting genotyping and transient elastography. 5 are no longer patients of the primary care clinic and attempts have been made to arrange onward referrals to Hepatology services in their new locations and 5 patients have died.

Conclusion Persons experiencing homelessness often have difficulty accessing healthcare. By facilitating access to Hepatology services tailored to their needs at a site where they access primary care and receive opiate substitution therapy, favourable SVR rates can be achieved with significant risk reduction.

\section{P034 IMPACT OF KAFTRIO ${ }^{\circledR}$ ON TACROLIMUS LEVELS AND LIVER GRAFT FUNCTION IN LIVER TRANSPLANT RECIPIENTS}

${ }^{1}$ Lindsay Smith*, ${ }^{2}$ Claire Kelly, ${ }^{1,3}$ Cathrine McKenzie, ${ }^{4}$ Felicity Perrin, 2,5 Jeremy S Nayagam, ${ }^{4}$ Ashley Thurlow, ${ }^{2}$ Deepak Joshi. ${ }^{1}$ Department of Pharmacy, King's College Hospital NHS Foundation Trust, London, UK; ' Institute of Liver Studies, King's College Hospital NHS Foundation Trust, London, UK; ${ }^{3}$ Institute of Cancer and Pharmacy, King's College London, London, UK; ${ }^{4}$ Department of Cystic Fibrosis, King's College Hospital, NHS Foundation Trust, London, UK; ${ }^{5}$ Department of Inflammation Biology, King's College London, London, UK

\subsection{6/gutjnl-2021-BASL.43}

Introduction Cystic fibrosis transmembrane conductance regulator (CFTR) modulators have been shown to increase lung function in Cystic Fibrosis (CF) patients who have two F508del mutations or one F508del combined with any other mutation. ${ }^{2}$ In non-transplant patients, trial data for liver function test (LFT) abnormality reported similar rates of transaminase elevation with CFTR modulators and placebo groups. ${ }^{1}$ There is limited data and experience of Kaftrio ${ }^{\circledR}$ in liver transplant (LT) recipients.

Methods Patients were identified through referrals to the liver transplant team for monitoring of their tacrolimus levels by the liver transplant multidisciplinary team (MDT). Prospective data was collected from electronic patient records. Our proto-

\begin{tabular}{|c|c|c|c|c|c|c|c|c|c|c|c|c|}
\hline \multirow[t]{2}{*}{$\begin{array}{l}\text { Patient } \\
\text { number }\end{array}$} & \multicolumn{2}{|c|}{ AST (IU/L) } & \multicolumn{2}{|c|}{ ALT (IU/L) } & \multicolumn{2}{|c|}{ ALP (IU/L) } & \multicolumn{2}{|c|}{$\begin{array}{l}\text { GGT (IU/ } \\
\text { L) }\end{array}$} & \multicolumn{2}{|c|}{$\begin{array}{l}\text { Billirubin } \\
\text { (IU/L) }\end{array}$} & \multicolumn{2}{|c|}{$\begin{array}{l}\text { Creatinine } \\
\text { (umol/L) }\end{array}$} \\
\hline & B & $\mathrm{R}$ & B & $\mathrm{R}$ & B & $\mathrm{R}$ & B & $\mathrm{R}$ & B & $R$ & B & $\mathrm{R}$ \\
\hline 1 & 19 & 25 & & & 76 & 84 & 18 & 20 & 6 & 17 & 68 & 79 \\
\hline 2 & 27 & 31 & & & 83 & 96 & 18 & 18 & 4 & 6 & 115 & 122 \\
\hline 3 & & & 12 & 17 & 92 & 123 & 45 & 85 & 8 & 8 & 87 & 82 \\
\hline 4 & 14 & & & 22 & 100 & 98 & 12 & & 8 & 9 & 82 & 64 \\
\hline 5 & & & 17 & 33 & 103 & 108 & & & 28 & 36 & 86 & 114 \\
\hline 6 & 21 & 16 & & & 90 & 70 & 12 & 12 & 5 & 15 & 60 & 62 \\
\hline 7 & 36 & 37 & & & 236 & 176 & 47 & 52 & 7 & 8 & 587 & 654 \\
\hline 8 & 44 & 62 & & & 158 & 250 & 37 & 76 & 12 & 13 & 80 & 89 \\
\hline
\end{tabular}

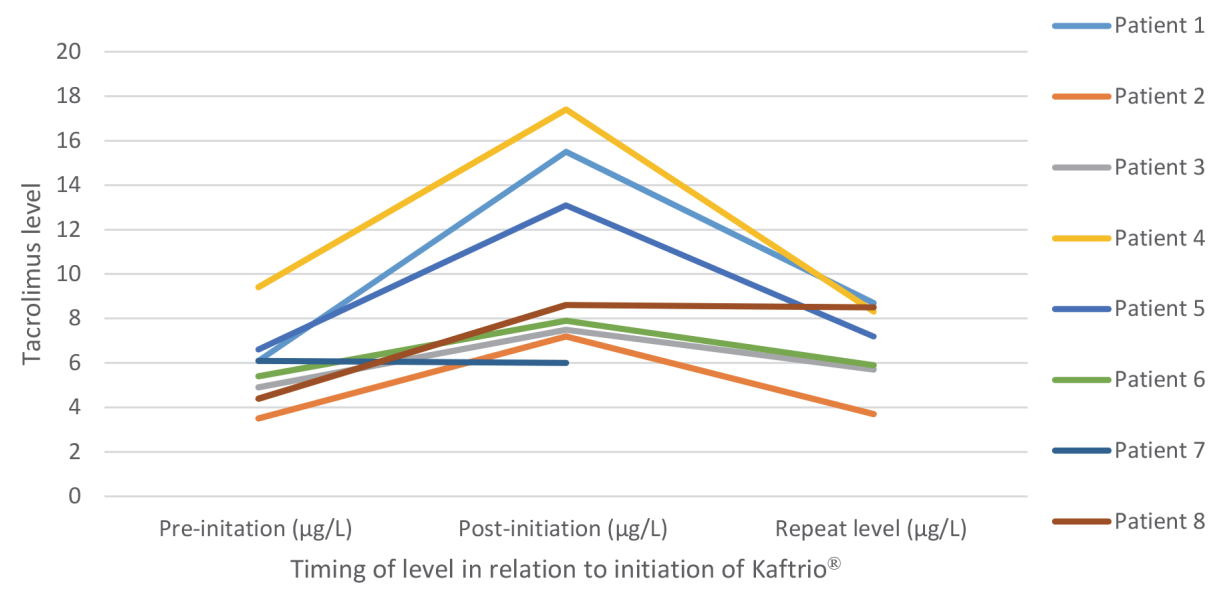

Abstract P034 Figure 1 The impact of Kaftrio ${ }^{\circledR}$ initiation on tacrolimus levels in 8 liver transplant recipients 